Rhea V. Samonte $\cdot$ Robert A Conte $\cdot$ Ram S. Verma

\title{
Molecular phylogenetics of the hominoid $Y$ chromosome
}

Received: January 13 / Accepted: April 7, 1998

\begin{abstract}
The Human Y-chromosome plays a central role in sex determination, and is composed of DNA sequences homologous to the Y-chromosome, families of Y-specific repetitive DNA sequences, and single copy sequences. We investigated the chromosomal location of Y-specific DNA sequences, in the chimpanzee (Pan troglodytes), gorilla (Gorilla gorilla), and orangutan (Pongo pygmaeus) by the fluorescence in situ hybridization (FISH) technique. The Yq subtelomeric DNA sequences (DYS427) have been observed to be intact at the presumed loci. Also, the amelogenin gene (AMELY, Yp11.2) revealed sequence homology and positional conservation in the higher primates, except in gorilla where positional divergence was observed.
\end{abstract}

Key words Chimpanzee $\cdot$ Gorilla $\cdot$ Orangutan $\cdot$ Human Ychromosome $\cdot$ Amelogenin gene $\cdot$ Telomeric sequences · Evolution

\section{Introduction}

The human Y-chromosome is one of the smallest human chromosomes, with an estimated size of 60 million base pairs (Hammer 1995). The evolutionary divergence of the hominoid lineage of human, chimpanzee, gorilla and orangutan suggests that the human Y-chromosome is of a recent event (Burrows and Ryder 1997). We used human Yq subtelomeric DNA sequence (DYS427) and the

R.V. Samonte $\cdot$ R.A. Conte $\cdot$ R.S. Verma $(\bowtie)^{1}$

Division of Genetics, The Long Island College Hospital - SUNY

Health Science Center, Brooklyn, USA

Tel. +1-718-745-0443; Fax +1-718-745-3966

\section{Present address:}

${ }^{1}$ Division of Genetics, Long Island College Hospital, 339 Hicks St, Brooklyn, N.Y. 11201-5514, USA

* Present address:

Natural Science Research Institute, University of the Philippines,

Diliman, Quezon, Philippines, 1101 amelogenin DNA sequence (AMELY) probes to elucidate the positional conservation or divergence.

\section{Materials and methods}

Chromosomes were prepared from fibroblast cell lines of chimpanzee (AGO 6939A, Pan troglodytes; PTR) and orangutan (AGO 5252, Pongo pygmaeus; PPY) obtained from the Coriell Institute for Medical Research, Camden, NY, USA, while chromosomes were obtained from a lymphoblast cell line of gorilla (CRL 1854, Gorilla gorilla; GGO) from the American Type Tissue Culture Collection (Rockville, MD, USA). Human chromosomes were prepared from phytohemagglutinin (PHA) stimulated lymphocyte cultures of normal individuals. Harvesting of cell cultures was performed using standard protocols (Verma and Babu, 1995). The fluorescence in situ hybridization (FISH) technique was used, as described by Lichter et al. (1991). The tel Yq DNA probe (DYS427) and QuintEssential Y-specific DNA probe (AMELY) (Oncor, Gaithersburg, MD, USA) were used.

\section{Results and discussion}

Localization of Y-specific DNA sequences was observed by hybridization of human chromosome Y-derived DNA probes to chromosomes of the chimpanzee, gorilla, and orangutan. Hybridization signals were observed using the human Y chromosome-specific subtelomeric DNA sequence probe (DYS427) and the Quint-Essential amelogenin DNA sequence probe (AMELY). The Yq subtelomeric DNA sequence has been conserved in the higher primates (PTR, Yq11-qter; GGO, Yq12-qter; and PPY, Yq11-qter) (Fig. 1) although such sequences are known to be highly polymorphic and unstable in the human (Brown et al. 1990; Pezzolo et al. 1993). The amelogenin gene (AMELY) has also been conserved at the equivalent 
Fig. 1 A Hybridization signals observed on the chromosomes of (a) humans, $(b)$ chimpanzee, (c) gorilla, and $(d)$ orangutan, using the human Yq subtelomeric DNA sequence probe (DYS427). B Representative idiogram showing the location of the hybridization signals. $A r$ rows have been assigned arbitrarily (ISCN, 1985). HSA, human; PTR, chimpanzee; GGO, gorilla; $P P Y$, orangutan
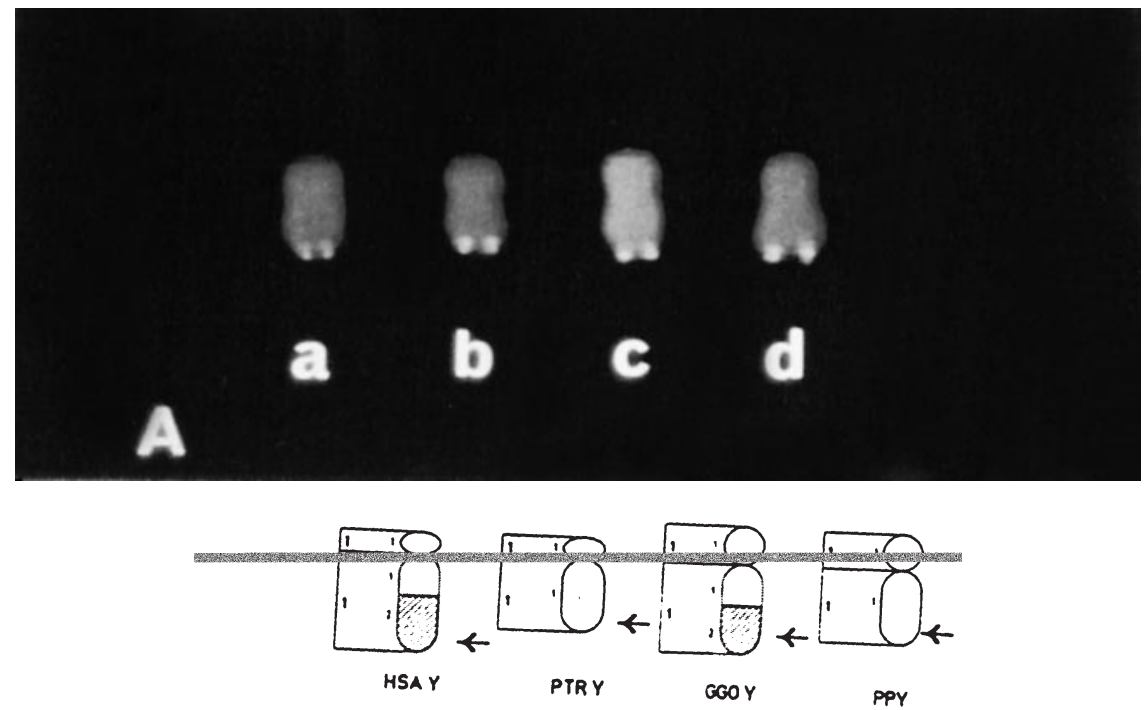

B

Fig. 2 A The amelogenin DNA sequence probe (AMELY) specific for human chromosome Yp11.2 was hybridized to the chromosomes of $(a)$ humans, $(b)$ chimpanzee, $(c)$ gorilla, and $(d)$ orangutan. A shifting of the locus was observed in the gorilla chromosomes. B Representative idiogram showing the location of the hybridization signals. Arrows have been assigned arbitrarily (ISCN, 1985)

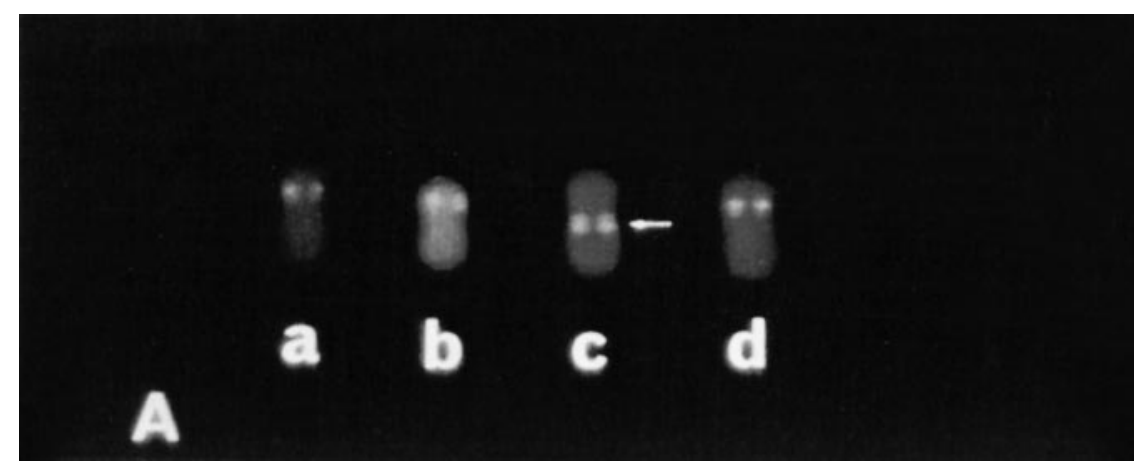

Yp11 chromosome regions, although a shifting of this locus has been observed in the gorilla, wherein it has been localized in band PTR Yq11 (Fig. 2). The difference in the location may be attributed to a pericentric inversion in the gorilla.

Aside from our observation on the shifting of the AMELY locus, several Y-linked sequences have been shown to exibit an accelerated rate of evolution (Simmin et al. 1993). The conservation of unique DNA sequences on the great ape $\mathrm{Y}$ chromosomes indicates a greater stability in this region of the $\mathrm{Y}$ chromosome than has been previously described for most anonymous human Y chromosomal regions. The stability of these sequences on great ape $Y$ chromosomes seems remarkable, given that this region of the $Y$ does not undergo meiotic recombination and the sequences do not appear to encode genes for which positive selection might occur. Recently available loci-specific probes will serve as phylogenetic markers in describing the structural chromosomal changes in humans.
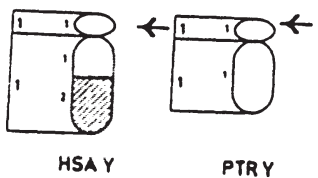

PTRY

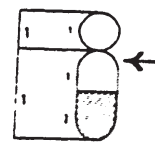

GGOY

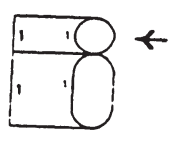

PpY

$B$

\section{References}

Brown WR, MacKinnon PJ, Villasante A, Spurr N, Buckle VJ, Dobson MJ (1990) Structure and polymorphism of human telomereassociated DNA. Cell 63: 119-132

Burrows W, Ryder OA (1997) Y-chromosome variation in great apes. Nature 385: $125-126$

Hammer MF (1995) A recent common ancestry for human Ychromosome. Nature 378: 376-380

ISCN (1985) An international system for human cytogenetic nomenclature. S. Karger, Basel

Lichter P, Boyle AL, Cremer T, Ward DC (1991) Analysis of genes and chromosomes by non-isotopic in situ hybridization. Genet Anal Tech Appl 8: 24-35

Pezzolo A, Gimelli G, Coehn A, Lavaggetto A, Romano C, Fogu G, Zuffardi O (1993) Presence of telomeric and sutelomeric sequences at the fusion point of ring chromosomes indicates that the ring syndrome is caused by ring instability. Hum Genet 92: 23-27

Simmin LC, Chang H-J, Li W-H (1993) Male driven evolution of DNA sequences. Nature 362: 745-747

Verma RS, Babu A (1995) Human chromosomes: Principles and techniques. McGraw-Hill, New York 